\title{
SLS-irritated human skin shows no correlation between degree of proliferation and TEWL increase
}

\begin{abstract}
It is well known that cutaneous irritants influence epidermal proliferation but the pathogenesis is poorly understood. Recent investigations have shown that the skin barrier integrity influences the proliferation of the basal keratinocytes. Our question was whether the proliferating activity of keratinocytes is indeed regulated by the degree of skin barrier damage or by a direct toxic action of the irritant on the keratinocytes. Therefore various degrees of skin irritation were induced by the application of $0.1 \%, 0.5 \%$ and $2 \%$ sodium lauryl sulphate (SLS) solution to the forearm skin of six healthy volunteers. This experiment was performed to evaluate the relationship between SLS concentration and epidermal proliferation. In a second experiment another 14 volunteers were treated with a single SLS concentration $(0.5 \%)$ to look for interindividual differences in the patterns of skin reaction and susceptibility to the irritant. Skin barrier function was evaluated by measurements of transepidermal water loss (TEWL) before and after irritation. Punch biopsies were taken after $96 \mathrm{~h}$ from exposed areas and from unexposed normal skin. Dividing keratinocytes were identified immunocytochemically using three different monoclonal antibodies: PCNA, MIB 1 and KiS1. Exposure to SLS resulted in concentrationdependent increases in both TEWL and epidermal proliferation. However, no significant correlation could be found between the degree of hyperproliferation and the TEWL changes. The results suggest that epidermal
\end{abstract}

Supported by a grant from the Deutsche Forschungsgemeinschaft (DFG Wi 879/3-2)

J. Welzel $(\bowtie) \cdot$ C. Metker · H. H. Wolff

Klinik für Dermatologie und Venerologie,

Medizinische Universität zu Lübeck, Ratzeburger Allee 160, D-23538 Lübeck, Germany

Tel.: +49-451-500 2515; Fax: +49-451-500 2981

K.-P. Wilhelm

proDERM, Industriestraße 1,

22869 Schenefeld/Hamburg, Germany proliferation is modulated by a direct interaction of the surfactant with the keratinocytes and/or by release of mediators rather than the consequence of a barrier disturbance.

Key words Skin barrier function - Proliferation · Sodium lauryl sulphate $\cdot$ Keratinocytes $\cdot$ Transepidermal water loss

\section{Introduction}

Experimental work in the field of irritant contact dermatitis has been increasing because the pathogenetic pathways are far from being completely understood.

The influence of the widely used anionic surfactant sodium lauryl sulphate (SLS) on human skin has been reported in several studies. On the one hand its potential to disrupt the skin barrier integrity as represented by the stratum corneum has been described [1, 3, 19, 22]. Agner and Serup [2] and Wilhelm et al. [20] found a linear relationship between the dose of SLS and the skin response as evaluated by noninvasive measurements, among others transepidermal water loss (TEWL). They proved measurement of TEWL to be the most sensitive method for quantification of the skin reaction to SLS. Disturbed organization of the lipid bilayers [13] and the extraction of lipids from the stratum corneum $[5,7,9,10]$ are thought to be the causes of the loss of barrier function.

On the other hand, it is well documented that topical application of SLS induces an increase in the mitotic activity of basal keratinocytes. Fisher and Maibach [8] have shown that SLS in a range of concentrations causes a rise in the mitotic activity of basal epidermal cells in human skin. It has been demonstrated that irritation increases stratum corneum turnover [21]. SLS induces not only changes in the stratum corneum but also penetrates into the deeper parts of the viable epidermis [7]. This results in alterations to the keratinocytes with damage to the cell membranes [23] and the expression of various cytokines which are able to influence and modify the normal growth 
patterns of the keratinocytes [14, 24, 26, 27]. Willis et al. [25] investigated the effects of different chemical irritants on the density of proliferating keratinocytes and also demonstrated a significant increase in dividing cells after irritation with SLS. However, Willis and coworkers did not find a relationship between the density of proliferating cells and the visually assessed intensity of inflammation.

Proksch et al. [16] have demonstrated that the increase in DNA synthesis is linearly correlated with the degree of disturbance of the barrier function after tape stripping using a hairless mouse model. They underlined that the stratum corneum plays a role in the control of epidermal proliferation.

Because of these conflicting findings, it was our aim to investigate the effect of SLS-induced damage to the barrier function on the proliferation state of keratinocytes in human skin. In a first experiment different concentrations of the irritant were applied to look for dose-dependent effects on barrier disturbance and proliferation. A second experiment was performed with a single dose of SLS to investigate individual differences in susceptibility to the irritant. Therefore, the degree of TEWL increase was compared with the resulting proliferating activity to evaluate whether the TEWL increase can predict the degree of proliferation.

\section{Materials and methods}

Patch testing

A group of 20 healthy volunteers participated in the study (10 female and 10 male, median age 31 years, range $24-54$ years). Informed consent was obtained from all subjects, and the study was approved by the local ethical committee.

In the first experiment (experiment 1) six volunteers were treated with $100 \propto d$ of an aqueous solution of SLS at $0.1 \%, 0.5 \%$ and $2 \%$ (Merck, Düsseldorf, Germany) applied to the volar aspect of the forearm for $24 \mathrm{~h}$, using polypropylene chambers (diameter $19 \mathrm{~mm}$; Hill Top Research, Miamiville, Ohio) on Scanpore tape (Norgesplaster, Vennesla, Norway). Application of the SLS concentrations was rotated between the test areas to avoid anatomical selection bias. In the second experiment (experiment 2) only a single concentration of $0.5 \%$ SLS was applied to another 14 volunteers under the same conditions.

\section{TEWL measurement}

TEWL measurements were conducted on normal skin before and 24,48 and $96 \mathrm{~h}$ after SLS application using a tewameter (TM 210 , Courage \& Khazaka, Köln, Germany). The TEWL change after $48 \mathrm{~h}$ was entered into the statistical analysis. The values were corrected for the actual skin temperature. All volunteers rested for at least 15 min under laboratory conditions with a constant room temperature of $18-20^{\circ} \mathrm{C}$ and a relative humidity of $50-55 \%$.

\section{Biopsy}

In a preliminary study we investigated the kinetics of proliferation after irritation to determine the optimal time-point for taking the biopsies. Therefore, six volunteers were treated with $0.5 \%$ SLS applied to healthy skin next to skin tumours before planned surgical excision. Biopsies were taken after 1, 2, 3, 4, 7 or 9 days. The proliferation showed a high interindividual and time-dependent vari- ability. The highest activity was found in the specimen taken 4 days after irritation. Therefore, we decided to take the punch biopsies $(4 \mathrm{~mm})$ from unirritated normal skin and from each patch tested area $96 \mathrm{~h}$ after irritation induction, using $1 \%$ mepivacaine as local anaesthetic. In experiment 1 , four biopsies were taken from each volunteer, and in experiment 2 , two biopsies were taken.

\section{Immunocytochemistry}

The paraffin-embedded tissue was cut into sections of thickness $4 \mathrm{~cm}$. Two sections per proliferating marker per skin sample were stained with PCNA (DAKO, Glostrup, Denmark) diluted 1:50, with MIB 1 (Dianova, Hamburg, Germany) and KiS1 (kindly made available by the Institute of Pathology and Haematopathology, Kiel, Germany), both diluted $1: 10$. The antibody/antigen reaction was visualized using the alkaline phosphatase antialkaline phosphatase technique. The counterstaining was performed with Mayer's haematoxylin.

\section{Microscopy}

The sections were examined at a total magnification of $\cdot 400$. All of the PCNA-, MIB 1- and KiS1-positive keratinocytes in each section were counted by hand with the aid of a haemocytometer. This was followed by a total count of all basal keratinocytes including stained and unstained cells. Each section was evaluated twice and without knowledge of the treatment. A mean value for the number of proliferating keratinocytes per 100 basal cells for each biopsy and each antibody was then calculated by combining the results obtained from the four counts in the two sections.

\section{Statistical methods}

The increases in TEWL and proliferating activity in comparison with normal skin were analysed using Wilcoxon's signed ranks test for matched pairs. In experiment 1, statistical comparison was made between the mean values of the TEWL $48 \mathrm{~h}$ after irritation and also between the mean number of proliferating keratinocytes in each SLS concentration group using the Friedman rank variance analysis with consecutive Wilcoxon/Wilcox comparisons. This statistical method allows the comparison of the degree of increase between different groups. In experiment 2, the correlation between TEWL and the density of dividing keratinocytes was investigated using Spearman's rank coefficient of correlation [18].

\section{Results}

\section{Experiment 1}

Irritation with SLS resulted in dose-dependent changes in TEWL and proliferating activity. The highest TEWL values were reached $48 \mathrm{~h}$ after irritation with $2 \%$ SLS in four of the six subjects and with $0.5 \%$ SLS in three subjects (Fig. 1). The proliferating activity of basal keratinocytes also increased after irritation. This was observed with all three proliferation markers (Fig. 2) with some differences in the number of positive cells and the specificity of staining. MIB 1 stained the highest number of keratinocytes in normal skin and in irritated skin. PCNA and KiS1 showed lower numbers of positive cells and a stronger background staining. The increases in both TEWL and proliferation showed a correlation with the SLS concentration (Fig. 3). The highest increase was induced by the $2 \%$ SLS 


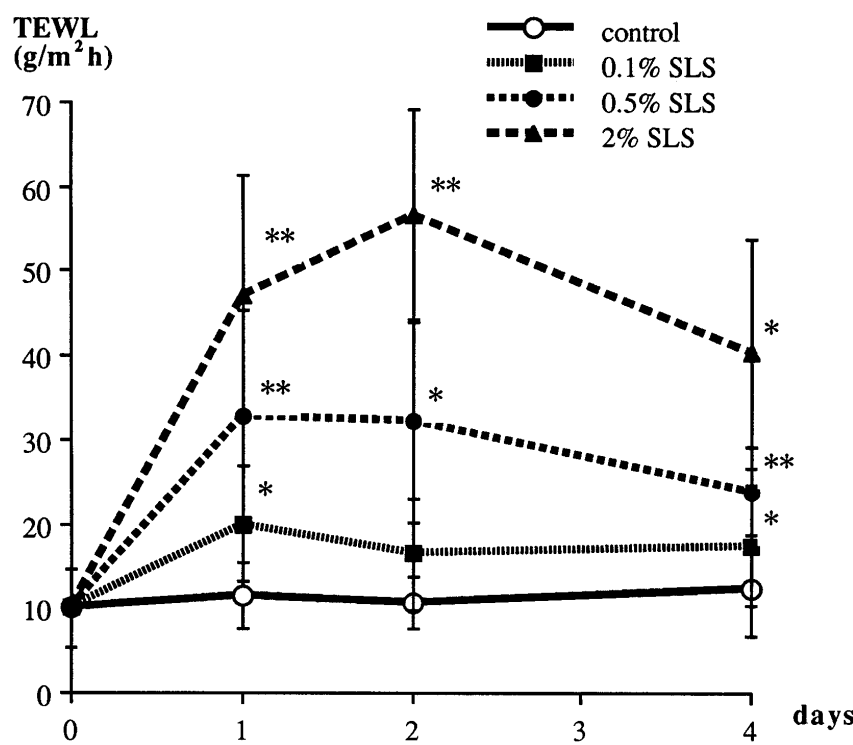

Fig. 1 Changes in TEWL $\left(\mathrm{g} / \mathrm{m}^{2}\right.$ per $\left.\mathrm{h}\right)$ before irritation and after 24,48 and $96 \mathrm{~h}$, irritation with $0.1 \%, 0.5 \%$ and $2 \%$ SLS. Values are means \pm standard deviation $(* P<0.05, * * P<0.001)$

\section{proliferation}

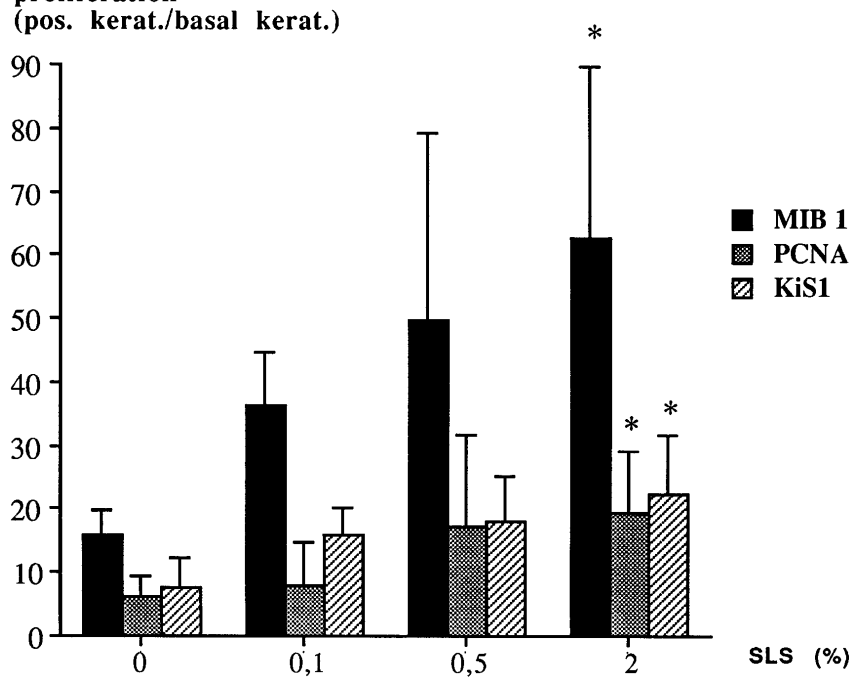

Fig. 2 Changes in proliferation for MIB 1, KiS1 and PCNA $96 \mathrm{~h}$ after irritation with $0.1 \%, 0.5 \%$ and $2 \%$ SLS (positive keratinocytes $/ 100$ basal keratinocytes). Values are means \pm standard deviation $(* P<0.05, * * P<0.001)$

concentration. The mean values of TEWL and the mean number of proliferating keratinocytes in each SLS concentration group are given in Table 1. Significant increases in TEWL after irritation occurred in response to $0.5 \%$ and $2 \%$ SLS $(P<0.001)$ whereas only the increase in proliferating activity in response to $2 \%$ SLS was statistically significant $(P<0.05)$ using the Wilcoxon's signed ranks test.

\section{proliferation \\ (pos. kerat./basal kerat.) \\ TEWL $\left(\mathrm{g} / \mathrm{m}^{2} \mathrm{~h}\right)$}

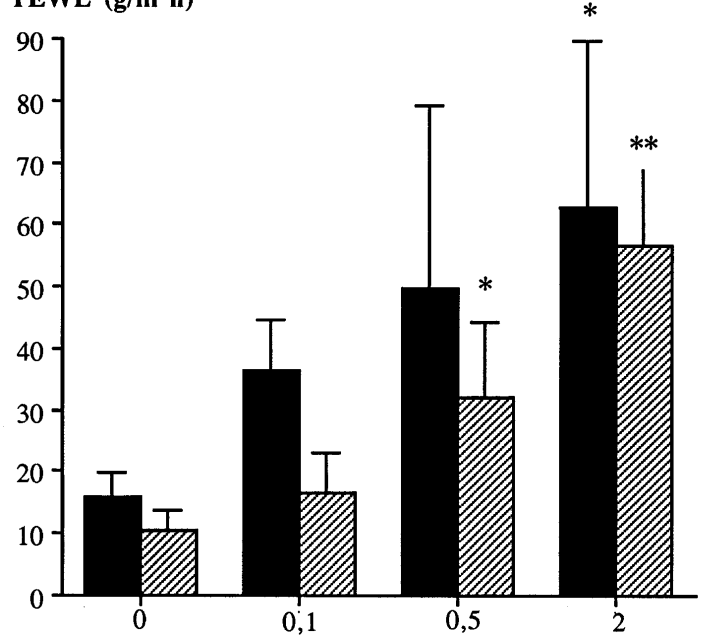

MIB 1

TEWL

Fig. 3 Dose-dependent increases in TEWL $\left(\mathrm{g} / \mathrm{m}^{2}\right.$ per h) $48 \mathrm{~h}$ after irritation and proliferation (MIB 1-positive keratinocytes/100 basal keratinocytes) $96 \mathrm{~h}$ after irritation with $0.1 \%, 0.5 \%$ and $2 \%$ SLS Values are means \pm standard deviation $(* P<0.05, * * P<0.001)$

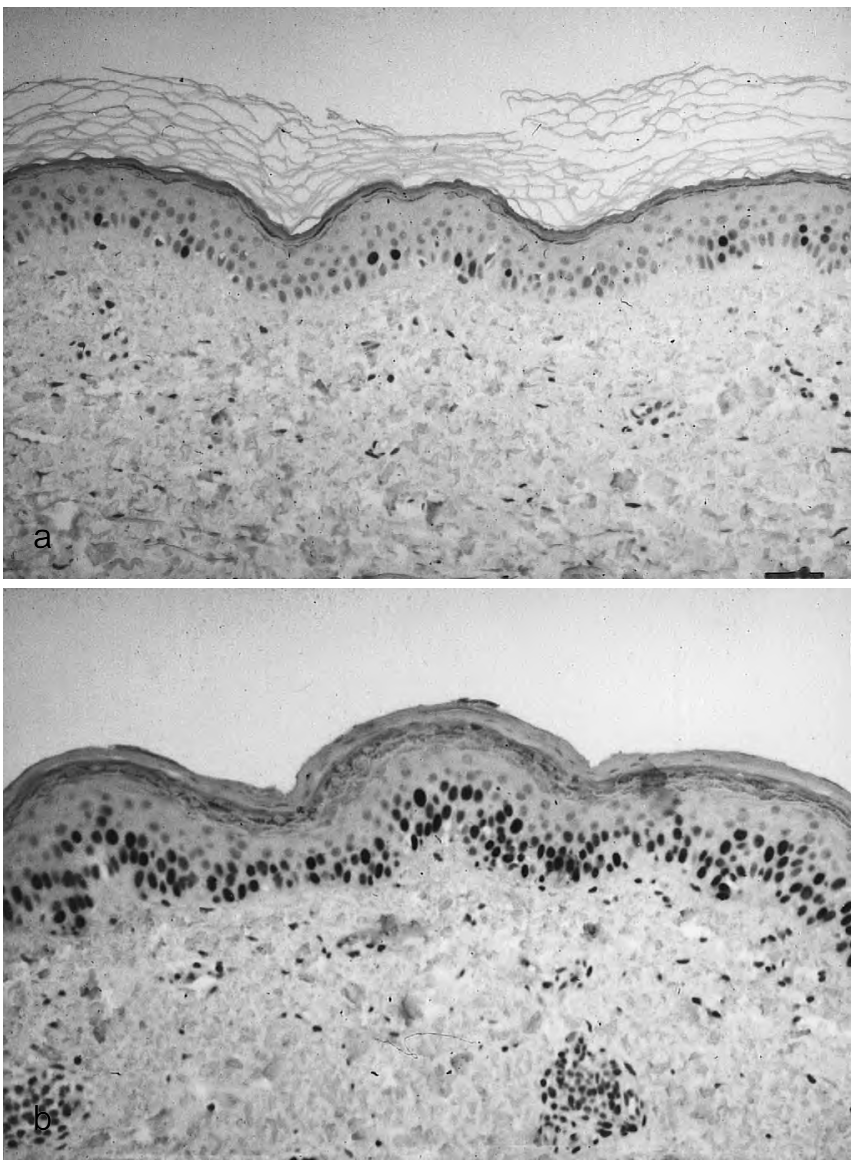

Fig. 4 a Section of normal skin immunolabelled with MIB 1 demonstrating the density of proliferating keratinocytes within the basal layers of the epidermis. b Irritation with a $0.5 \%$ aqueous solution of SLS caused a marked increase in the density of dividing cells after $96 \mathrm{~h}$ 


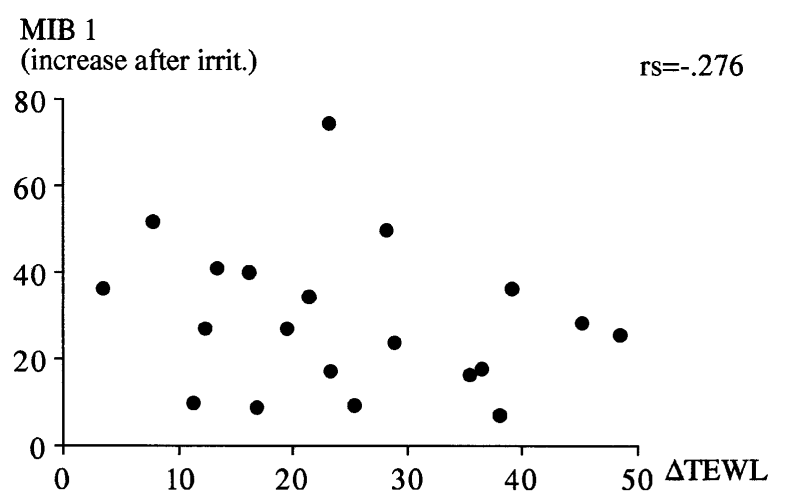

\section{PCNA}

(increase after irrit.)

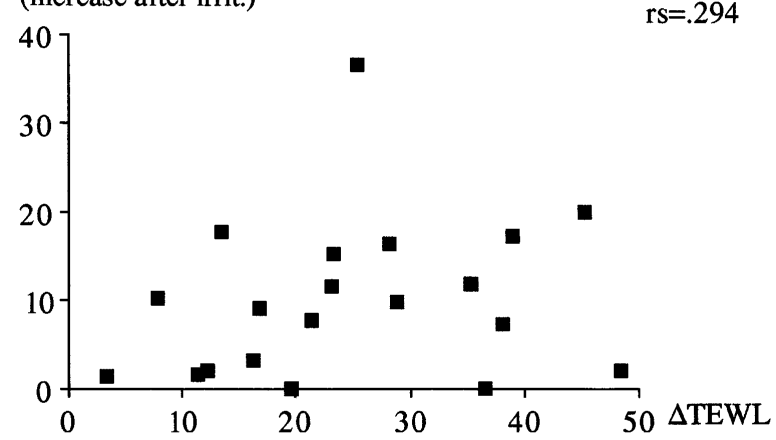

KiS1

(increase after irrit.)

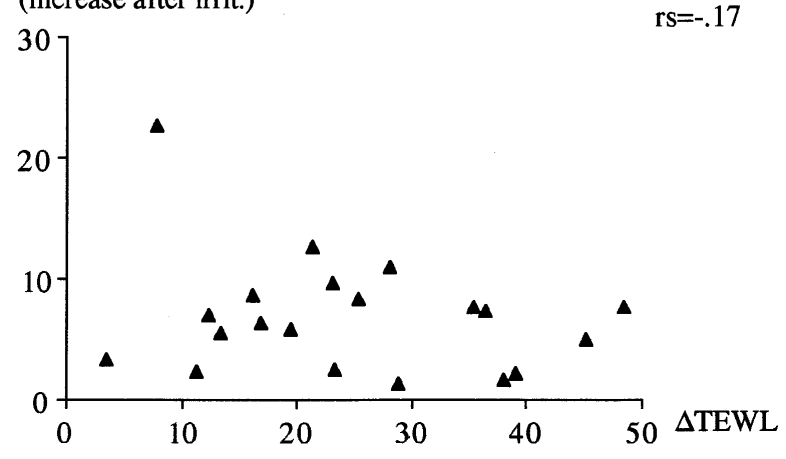

Fig. 5 Results of Spearman's rank correlation coefficient test. Scattergrams show no significant correlation between the degree of barrier perturbation (difference between TEWL after $48 \mathrm{~h}$ and TEWL of normal skin) and the extent of the increase in epidermal proliferation (difference between proliferation rate after irritation with $0.5 \%$ SLS and proliferation rate of normal skin) for proliferation markers MIB 1, KiS1 and PCNA

\section{Experiment 2}

As in experiment 1 , irritation with $0.5 \%$ SLS caused significant increases in TEWL and proliferating activity in comparison with unexposed skin (Wilcoxon test for matched pairs, $P<0.001)$. A high interindividual variability of the degree of barrier disturbance after identical irritation was observed. The TEWL values ranged from 15 to $66 \mathrm{~g} / \mathrm{m}^{2}$ per h after $48 \mathrm{~h}$. Figure 4 shows sections of normal (a) and irritated (b) skin immunolabelled with MIB 1. The scattergrams in Fig. 5 demonstrate the change
Table 1 Mean values ( \pm standard deviation, $n=6$ ) of TEWL after $48 \mathrm{~h}\left(\mathrm{~g} / \mathrm{m}^{2}\right.$ per $\left.\mathrm{h}\right)$ and proliferating keratinocytes (positive keratinocytes/100 basal cells) $96 \mathrm{~h}$ after irritation with $0.1 \%, 0.5 \%$ and $2 \%$ aqueous solution of SLS and in unirritated skin. $(* P<0.05$, $* * P<0.001$, vs normal skin)

\begin{tabular}{|c|c|c|c|c|}
\hline & \multicolumn{2}{|c|}{ Normal skin $0.1 \%$ SLS } & $0.5 \%$ SLS & $2 \% \mathrm{SLS}$ \\
\hline MIB 1 & $16.0 \pm 3.8$ & $36.5 \pm 8.2$ & $49.6 \pm 29.5$ & $62.5 \pm 27.3^{*}$ \\
\hline KiS1 & $7.5 \pm 4.8$ & $15.7 \pm 4.6$ & $18.0 \pm 7.2$ & $22.3 \pm 9.3 *$ \\
\hline PCNA & $6.1 \pm 3.3$ & $8.1 \pm 6.6$ & $17.4 \pm 14.1$ & $19.4 \pm 9.8^{*}$ \\
\hline TEWL & $10.6 \pm 3.1$ & $16.4 \pm 6.7$ & $32.1 \pm 12.0^{*}$ & $56.5 \pm 12.6^{* *}$ \\
\hline
\end{tabular}

in the density of proliferating keratinocytes in relation to the increase in TEWL. The conditions varied slightly between the antibodies used. However, Spearman's rank coefficient of correlation indicated that there was no linear correlation between the two parameters.

\section{Discussion}

The integrity of the stratum corneum is essential for normal skin barrier function. Several studies have shown that topical application of irritants leads to a disturbance of the stratum corneum with a subsequent TEWL increase due to disorganization of the lipid bilayers, removal of cholesterol and free fatty acids with a subsequent increase in lipid synthesis [15], and denaturation of keratin. Other groups have investigated the penetration of SLS and its interaction with keratinocytes in the deeper cell layers of the epidermis. There are several mechanisms believed to influence the kinetics of keratinocyte proliferation, e.g. the effects of cytokines, growth factors, calcium concentration and other mediators [14, 24, 26, 27].

Two distinct ways by which the irritant response modulates epidermal proliferation have been formulated: (a) direct interaction of the irritant with the keratinocytes and (b) an indirect mechanism via damage to the skin barrier. In our study, we confirmed that abnormal barrier function after a 24-h irritation was accompanied by epidermal hyperproliferation even 4 days after SLS application. Furthermore, in the experiment 1 we demonstrated that both the TEWL and the density of proliferating keratinocytes were related to the SLS dose (Table 1).

Proksch et al. [16] have postulated a direct link between barrier function and epidermal DNA synthesis in a hairless mouse model. However, we were not able to confirm this direct relationship between proliferation rate and barrier damage in our in vivo human study. It seems more likely that the proliferating activity occurring after irritation with $0.5 \%$ SLS in experiment 2 was rather a nonspecific response to irritation. We could not confirm that the degree of damage to the skin barrier is proportional to the increase in cell division rate. It appears that conclusions from animal experiments are not easily transferable to conditions in human skin.

There are differences between the model of SLS irritation and tape stripping which is said to be a mechanical ir- 
ritant influencing only the barrier. The TEWL increase after SLS irritation is not only due to extraction of lipids but is also a consequence of inflammation and spongiosis. Recent studies have shown that the mode of action of irritants is always more complex than only disturbing the stratum corneum. Denda et al. [6] have investigated the effects of repeated tape stripping and have found that it also leads to cytokine production in the viable epidermis. The results of the study are in accordance with our results that the hyperplasia is not directly linked to the water loss.

In our study the proliferating activity of the epidermis was determined using three different monoclonal antibodies. The cell counting conducted in our experiments was semiquantitative with known shortcomings. One was the variable expression of the antigen during the cell cycle with different staining intensities, which may have made it difficult to evaluate positively labelled cells. Comparing the results with the three proliferating markers, it is noteworthy that there were higher values for MIB 1 than for PCNA and KiS1. The monoclonal antibody MIB 1 is associated with all active phases of the cell cycle [4], whereas PCNA shows maximum staining of cells in the S phase [12], and KiS1 expression increases with progression through the cell cycle, and G1 cells exhibit a weaker labelling than $\mathrm{G} 2 / \mathrm{M}$ cells [11]. This indicates that the keratinocytes at our time-point of $96 \mathrm{~h}$ after irritation were in the later phases of the cell cycle in G2 and M phase as well as in G1 and S phase. Because of the more specific association of PCNA and KiS1 antibody with one of the cell cycle phases, it might be that the number of proliferating cells was underestimated. However, there are numerous sources of error, and we have to be careful in interpreting immunohistochemical data. Because of these problems we decided to use three different markers to increase the validity. In agreement with Rose et al. [17] we found MIB 1 to be the most suitable proliferating marker, not least because of its clear labelling pattern.

In conclusion, our results indicate that irritant-induced hyperproliferation of human epidermal keratinocytes is dose dependent. The degree of proliferation is modulated by a direct interaction of the irritant with the keratinocytes and/or by release of proliferation-regulating mediators, rather than the consequence of perturbation of the barrier function. The heterogeneous pattern of skin reaction in our study emphasizes the individual nature of susceptibility to irritants and shows that TEWL measurement cannot provide information about predictable changes of the human epidermal cell kinetics.

Acknowledgements We are very grateful to Mrs. I. Schaller for her expert technical assistance. We would like to thank our volunteers who made the study possible.

\section{References}

1. Agner T (1991) Basal transepidermal water loss, skin thickness, skin blood flow and skin colour in relation to sodium lauryl sulphate induced irritation in normal skin. Contact Dermatitis $25: 108-114$
2. Agner T, Serup J (1990) Sodium lauryl sulphate for irritant patch testing - a dose response study using bioengineering methods for determination of skin irritation. J Invest Dermatol $95: 543-547$

3. Agner T, Serup J, Handlos V, Batsberg W (1989) Different skin irritation abilities of different qualities of sodium lauryl sulphate. Contact Dermatitis $21: 184-188$

4. Cattoretti G, Becker MHG, Key G, Dochrow M, Schlüter C, Galle J, Gerdes J (1992) Monoclonal antibodies against recombinant parts of the Ki67 antigen (MIB 1 and MIB 3) detect proliferating cells in microwave processed formalin-fixed paraffin sections. J Pathol 168:357-363

5.Denda M, Koyama J, Namba R, Horii I (1994) Stratum corneum lipid morphology and transepidermal water loss in normal skin and surfactant induced scaly skin. Arch Dermatol Res 286:41-46

6. Denda M, Wood LC, Emami S, Calhoun C, Brown BE, Elias PM, Feingold KR (1996) The epidermal hyperplasia associated with repeated barrier disruption by acetone treatment or tape stripping cannot be attributed to increased water loss. Arch Dermatol Res 288:230-238

7.Fartasch M (1997) Ultrastructure of the epidermal barrier after irritation. Microsc Res Tech 37: 193-199

8. Fisher LB, Maibach HJ (1975) Effects of some irritants on human epidermal mitosis. Contact Dermatitis 1:273-276

9. Froebe CL, Simion FA, Rhein LD, Cagan RH, Kligman A (1990) Stratum corneum lipid removal by surfactants: relation to in vivo irritation. Dermatologica $181: 277-283$

10. Fulmer AW, Kramer GJ (1986) Stratum corneum lipid abnormalities in surfactant induced dry scaly skin. J Invest Dermatol $86: 598-602$

11. Kreipe H, Heidebrecht HJ, Hansen S, Röhlk W, Kubbies M, Wacker HH, Tiemann M, Radzun HJ, Parwaresch R (1993) A new proliferation-associated nuclear antigen detectable in paraffin-embedded tissues by the monoclonal antibody Ki-S1. Am J Pathol 142:3-9

12. Kurki P, Vanderlaan M, Dolbeare F, Gray J, Tan EM (1986) Expression of proliferating cell nuclear antigen (PCNA)/cyclin during cell cycle. Exp Cell Res 166:209-219

13. Leveque JL, DeRigal J, Sain Leger D, Billy D (1993) How does sodium lauryl sulphate alter the skin barrier function in man? A multiparametric approach. Skin Pharmacol 6:111-115

14. Nickoloff BJ, Naidu Y (1994) Perturbation of epidermal barrier function correlates with initiation of cytokine cascade in human skin. J Am Acad Dermatol 30:535-546

15. Proksch E (1992) Regulation der epidermalen Permeabilitätsbarriere durch Lipide und durch Hyperproliferation. Hautarzt $43: 331-338$

16. Proksch E, Feingold KR, Elias PM (1991) Barrier function regulates epidermal DNA-synthesis. J Clin Invest 87: 1668-1673

17. Rose DSC, Maddox PH, Brown DC (1994) Which proliferation markers for routine immunohistology? A comparison of five antibodies. J Clin Pathol 47:1010-1014

18. Sachs L (1992) Angewandte Statistik, 7th edn. Springer, Berlin Heidelberg New York

19. Widmer J, Elsner P, Burg G (1994) Skin irritant reactivity following experimental cumulative irritant contact dermatitis. Contact Dermatitis 30:35-39

20. Wilhelm KP, Surber C, Maibach HI (1989) Quantification of sodium lauryl sulfate irritant dermatitis in man: comparison of four techniques: skin color reflectance, transepidermal water loss, laser Doppler flow measurement and visual scores. Arch Dermatol Res 281:293-295

21. Wilhelm KP, Saunders JC, Maibach HI (1990) Increased stratum corneum turnover induced by subclinical irritant dermatitis. Br J Dermatol 122: 793-798

22. Wilhelm KP, Freitag G, Wolff HH (1994) Surfactant induced skin irritation and skin repair: evaluation of the acute human irritation model by noninvasive techniques. J Am Acad Dermatol 30:944-949 
23. Willis CM, Stephens CJM, Wilkinson JD (1989) Epidermal damage induced by irritants in man: a light and electron microscopic study. J Invest Dermatol 93:695-699

24. Willis CM, Stephens CJM, Wilkinson JD (1991) Selective expression of immune associated surface antigen by keratinocytes in irritant contact dermatitis. J Invest Dermatol 96:505-511

25. Willis CM, Stephens CJM, Wilkinson JD (1992) Differential effects of structurally unrelated chemical irritants on the density of proliferating keratinocytes in $48 \mathrm{~h}$ patch test reactions. J Invest Dermatol 99:449-453
26. Wilmer JL, Burleson PG, Kayama F, Kanno J, Luster MI (1994) Cytokine induction in human epidermal keratinocytes exposed to contact irritants and its relation to chemical-induced inflammation in mouse skin. J Invest Dermatol 102:915

27. Wood LC, Jackson SM, Elias PM, Grunfeld G, Feingold KR (1992) Cutaneous barrier perturbation stimulates cytokine production in the epidermis of mice. J Clin Invest 90:482-487 